\title{
DETERMINACIÓN DE LA CONCENTRACIÓN DE QUITOSANO EN POLVO POR VISCOSIMETRÍA CAPILAR
}

\author{
Agustín A. Rodríguez Llegado ${ }^{a^{*}}$, Jesús A. Cjuno H. ${ }^{\mathrm{b}}$
}

\begin{abstract}
RESUMEN
El objetivo de este trabajo ha sido desarrollar un procedimiento para la determinación de la concentración de quitosano en muestras en polvo, por viscosimetría capilar de Ostwald (capilar de 0,55 \pm 0,02 $\mathrm{mm}$ de diámetro interno). Se prepararon soluciones de quitosano patrón entre $461 \mathrm{mg} /$ litro hasta $9138 \mathrm{mg} /$ litro y pH 1,24 para todos los casos. Se realizaron también ensayos a temperaturas entre $20^{\circ}$ a $50^{\circ} \mathrm{C}$ observándose linealidad con la concentración hasta antes de 9138,36 mg/litro. La dependencia más importante fue la de viscosidad relativa $\left(\eta_{\mathrm{r}}\right)$ frente a la concentración (concordante con la ecuación de Poiseuille) que en el rango de 461 $\mathrm{mg} /$ litro a $2808 \mathrm{mg} /$ litro dio una dependencia lineal con $\mathrm{r}^{2}=0,995$, posibilitando el análisis de muestras para el contenido de quitosano con resultados consistentes y equiparables con los referenciales.
\end{abstract}

Palabras clave: quitosano, viscosidad, $\mathrm{pH}$, concentración.

\section{DETERMINATION OF THE CONCENTRATION OF CHITOSAN IN POWDER BY CAPILLARY VISCOSIMETRY}

\begin{abstract}
The objective of this work has been to develop a procedure for the determination of the concentration of chitosan in powder samples, by capillary viscosimetry of Ostwald (capillary of $0.55 \pm 0.02 \mathrm{~mm}$ internal diameter). Standard chitosan solutions between $461 \mathrm{mg} / \mathrm{liter}$ up to $9138 \mathrm{mg} /$ liter and $\mathrm{pH} 1.24$ were prepared for all cases. Tests were also carried out at temperatures between $20^{\circ}$ to $50^{\circ} \mathrm{C}$, observing linearity with the concentration until before $9138 \mathrm{mg} /$ liter. The most important dependence was the relative viscosity $\left(\eta_{\mathrm{r}}\right)$ versus the concentration (concordant with the Poiseuille equation) which in the range $461 \mathrm{mg} /$ liter to $2808 \mathrm{mg} /$ liter gave a linear dependence with $\mathrm{r}^{2}=0.995$, enabling the analysis of samples for the content of chitosan with consistent results and comparable with the referentials.
\end{abstract}

Key words: chitosan, viscosity, $\mathrm{pH}$, concentration.

\footnotetext{
a*Facultad de Química e Ingeniería Química, UNMSM. Av. Universitaria / Av. Germán Amézaga s/n Lima-1, Perú, agustinrllegado@gmail.com

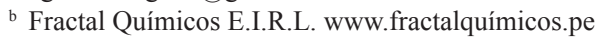




\section{INTRODUCCIÓN}

El quitosano es un poliaminosacárido lineal que deriva de la N-desacetilación por tratamiento alcalino de la quitina (constituyente del exoesqueleto de crustáceos, camarones o insectos). Esta N-desacetilación casi nunca se completa llegando en casos más óptimos este índice entre $75-95 \%$, cuya estructura química ${ }^{1,2,3}$ se aprecia en la figura 1 . La presencia de grupos amino libres a lo largo de la cadena permite que esta macromolécula (o biopolímero) se disuelva en disolventes ácidos diluidos debido a la protonación ${ }^{4,5}$ de estos grupos.

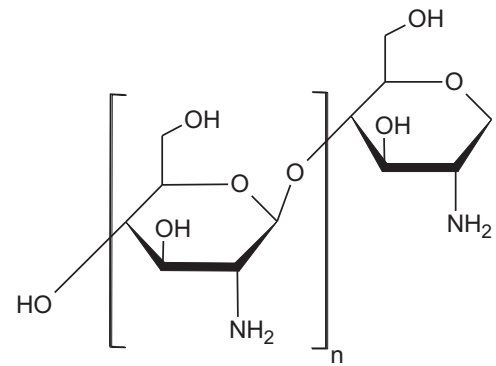

Figura 1. Quitosano ${ }^{1}$.

La presencia del quitosano (polímero) en el agua (solvente) genera como resultado un aumento de la viscosidad inclusive a muy bajas concentraciones. Este efecto depende de la concentración del polímero y la naturaleza del solvente. El agua permite que la macromolécula pueda hallarse desenrollada y alargada; es decir, es capaz de extender las cadenas lineales, así como desagregar ${ }^{6,7,8}$ dichas cadenas.

La viscosidad $(\eta)$ es la propiedad que caracteriza la resistencia que ofrece un fluido al flujo ${ }^{9}$. En las mediciones, la velocidad de flujo de un fluido en tubo capilar depende de las dimensiones de éste (radio y longitud), de la velocidad del fluido, de la diferencia de presión entre los extremos del tubo y de la temperatura. Para hallar la relación de estas magnitudes, se mide el flujo que pasa por cualquier punto del tubo capilar por unidad de tiempo. Finalmente, esta relación se demuestra en la fórmula de Poiseuille ${ }^{10}$ expresada en la ecuación 1.

$$
v=\frac{\pi P r^{4}}{8 \eta l}
$$

Donde $\mathrm{v}$ es la velocidad de flujo; $P$, presión que ejerce el fluido dentro del capilar; $r$, radio transversal interno del capilar; $\eta$, la viscosidad del fluido y $l$, longitud del capilar.

Despejando la ecuación de Poiseuille se tiene la ecuación 2.

$$
\eta=\frac{\pi P r^{4}}{8 v l}
$$




$$
\frac{\eta_{\text {solución }}}{\eta_{\text {solvente }}}=\frac{\left(\frac{\pi P_{\text {solución }} r^{4}}{8 \frac{V_{\text {solución }} l}{t_{\text {solución }}}}\right)}{\left(\frac{\pi P_{\text {solvente }} r^{4}}{8 \frac{V_{\text {solvente }}}{t_{\text {solvente }}}}\right)}=\frac{P_{\text {solución }} t_{\text {solución }}}{P_{\text {solvente }} t_{\text {solvente }}}
$$

Al considerar la presión, $P$ como el producto de la altura, $h$ del tubo capilar; la densidad del fluido, $\rho$ y la fuerza de gravedad, $g$ debido a que son constantes la altura del capilar y la fuerza de gravedad. Para soluciones diluidas no se presentan diferencias significativas de la densidad respecto al solvente, por lo que se halla la viscosidad relativa.

La viscosidad relativa $\left(\eta_{\mathrm{r}}\right)$ es la relación entre la viscosidad de la solución y la viscosidad del solvente ${ }^{10}$. Ambas viscosidades se miden en un viscosímetro capilar de precisión. La viscosidad relativa se define con la ecuación (4).

$$
\eta_{r}=\frac{\eta_{\text {solución }}}{\eta_{\text {solvente }}}=\frac{h \rho_{\text {solución }} g t_{\text {solución }}}{h \rho_{\text {solvente }} g t_{\text {solvente }}}=\frac{t_{\text {solución }}}{t_{\text {solvente }}}
$$

La viscosidad relativa se determina midiendo los tiempos de flujo del solvente y de las soluciones diluidas. La viscosidad relativa presenta una dependencia frente a la concentración según la ecuación 5 .

$$
\eta_{r}=\frac{\eta_{\text {solución }}}{\eta_{\text {solvente }}}=1+[\eta] C+K C^{2}+\cdots
$$

Donde $[\eta]$ y $K$ son constantes. Para $C=0, \eta_{\mathrm{r}}=1$ ya que $\eta_{\text {solución }}=\eta_{\text {solvente }}$; para bajas concentraciones (soluciones diluidas) se presenta una dependencia lineal mientras que a mayores concentraciones la dependencia es parabólica. La unidad física de viscosidad es el pascal por segundo (Pa.s) según el Sistema Internacional (S.I.), también se emplea como unidad el poise $(1 \mathrm{P}=1 \mathrm{~g} / \mathrm{cm} . \mathrm{s}=0,1 \mathrm{~Pa} . \mathrm{s})$, pero para fines de cálculo se emplea su submúltiplo el centipoise $\left(1 \mathrm{cP}=10^{-3} \mathrm{~Pa} . \mathrm{s}\right)$ debido a que el agua tiene una viscosidad de $1,002 \mathrm{cP}$ a $20^{\circ} \mathrm{C}$.

\section{PARTE EXPERIMENTAL}

\section{Reactivos}

Quitosano en polvo (patrón) grado alimentario de 90,3\% de pureza, solución de ácido clorhídrico $(\mathrm{HCl}) 0,1 \mathrm{~N}$, agua desionizada y etanol al $96 \% \mathrm{v} / \mathrm{v}$ (empleado para la limpieza del viscosímetro de Ostwald después de las mediciones, así como para el resto del material de vidrio). 


\section{Aparatos y materiales}

Balanza analítica electrónica digital $\pm 0,1 \mathrm{mg}$ M-Power-Sartorius, $\mathrm{pH} / \mathrm{mV}$-metro con microprocesador PHS-25CW-TKR (calibrado con buffers $\mathrm{pH} 4,00$ y $\mathrm{pH}$ 6,86 y protocolos normalizados), agitador magnético de 230V-Fisher Scientific, cronómetro $\pm 0,01$ seg. F-201 WA-1A-Casio, viscosímetro de Ostwald (bulbo superior de 6,2 mL; bulbo inferior de $9 \mathrm{~mL}$; $\phi_{\text {interno }}=0,55 \pm 0,02 \mathrm{~mm}$, termómetro de mercurio $\pm 0,1^{\circ} \mathrm{C}\left(-10\right.$ a $\left.100^{\circ} \mathrm{C}\right)$.

3. Muestras. Se prepararon soluciones de quitosano a concentraciones desde $461 \mathrm{mg} /$

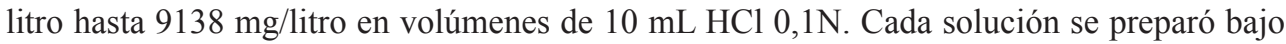
agitación magnética durante 20 minutos. Culminadas las disoluciones en medio ácido se tomaron lecturas de $\mathrm{pH}$. Estas lecturas tuvieron por objetivo corroborar que el polímero se encuentre en forma iónica ${ }^{11}$, mas no formando sales, lo cual es un estado idóneo para el estudio central realizado. Las condiciones de preparación quedan especificadas en la tabla 1.

Tabla 1. Condiciones de preparación de soluciones de quitosano.

\begin{tabular}{ccc}
\hline $\begin{array}{c}\text { Concentración } \\
(\mathbf{m g} / \text { litro })\end{array}$ & $\mathbf{W}_{\text {quitosano }}(\mathbf{g})$ & $\mathbf{p H}$ \\
\hline 0 & ------- & 1,20 \\
461 & 0,0051 & 1,22 \\
948 & 0,0105 & 1,25 \\
1355 & 0,0150 & 1,22 \\
1851 & 0,0205 & 1,20 \\
2294 & 0,0254 & 1,23 \\
2808 & 0,0311 & 1,23 \\
4614 & 0,0511 & 1,25 \\
6782 & 0,0751 & 1,27 \\
9138 & 0,1012 & 1,27 \\
\hline $\mathrm{W}=$ peso & &
\end{tabular}

4. Determinación de viscosidad. El equipo de determinación de viscosidad se muestra la figura 2. Se procedió a medir los tiempos de flujo de las soluciones de la tabla 1 , a $20^{\circ} \mathrm{C}$, $30^{\circ} \mathrm{C}, 40^{\circ} \mathrm{C}$ y $50^{\circ} \mathrm{C}\left( \pm 0,1^{\circ} \mathrm{C}\right)$. Los tiempos de flujo fueron tomados por quintuplicado después de esperar cuatro minutos luego de alcanzar el equilibrio térmico. 


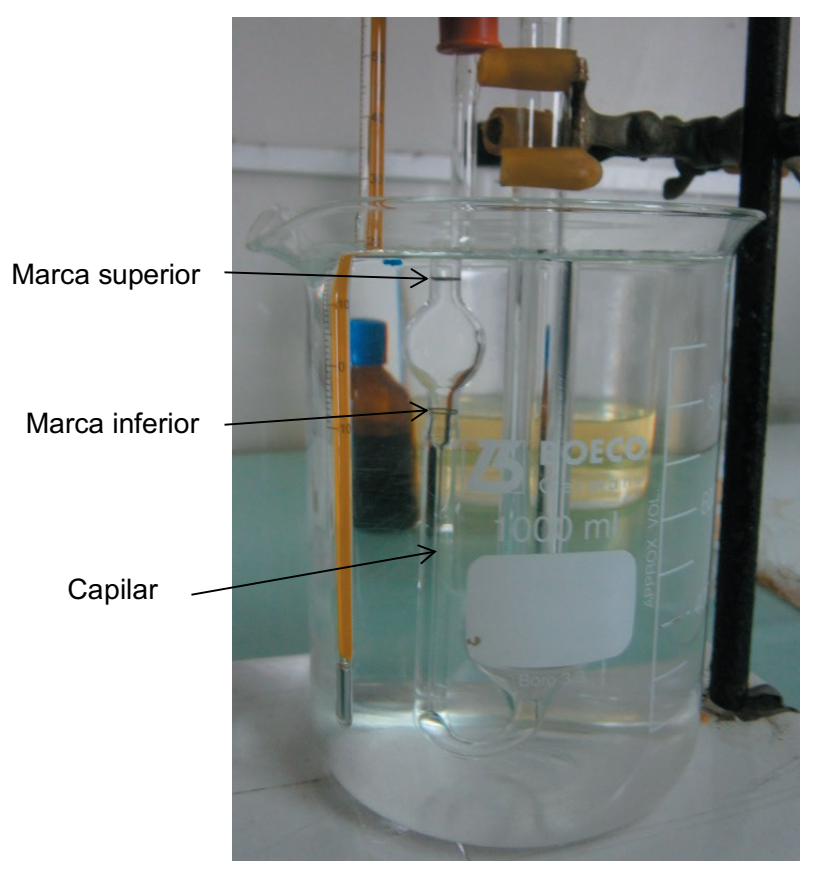

Figura 2. Equipo de determinación de la viscosidad. Se pueden ver las dos marcas, arriba y debajo del bulbo (marcas) así como el capilar de $0,55 \mathrm{~mm}$ de diámetro. Fuente: Fotografía del autor.

\section{RESULTADOS Y DISCUSIÓN}

En la figura 3 se muestra la gráfica de influencia de la temperatura sobre la viscosidad relativa de las soluciones de quitosano. Esta influencia ha permitido ver el comportamiento de interacción molecular entre el polímero catiónico y el ácido fuerte diluido en el rango de temperatura entre 20 a $50^{\circ} \mathrm{C}^{12,13,14}$. Se observó que existe un comportamiento lineal frente a la concentración hasta $9138 \mathrm{mg} /$ litro. 


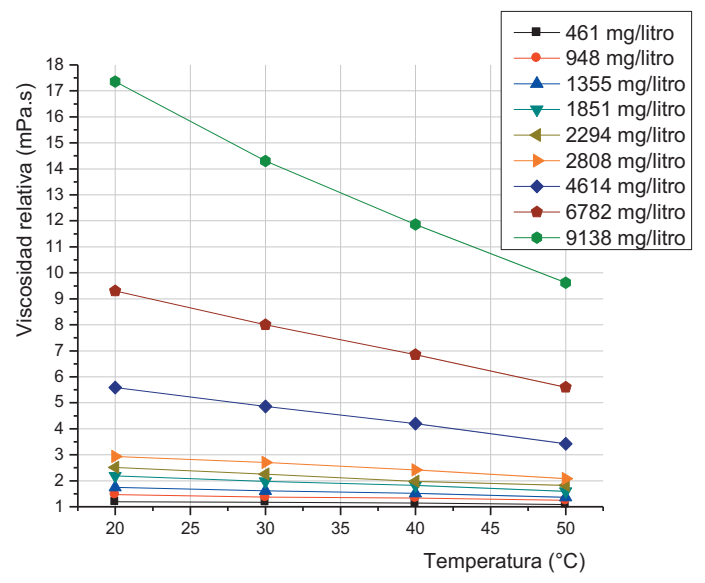

Figura 3. Influencia de la temperatura de equilibrio del sistema sobre la viscosidad relativa soluciones de quitosano.

De la figura 3, se tomaron en cuenta por criterios de norma internacional (AOAC) los valores de viscosidad a $20^{\circ} \mathrm{C}$. Es así que se puede evidenciar una tendencia de comportamiento lineal ${ }^{16,17,18}$ entre las concentraciones y las viscosidades hasta $2808 \mathrm{mg} /$ litro mostrados en la tabla 2 y figura 4 . Es de notar también que a viscosidades superiores a $2808 \mathrm{mg} /$ litro la dependencia de temperatura con la viscosidad tiene una tendencia parabólica (figura 4).

Tabla 2. Valores de coeficientes de viscosidad de soluciones de quitosano a $20^{\circ} \mathrm{C}$.

\begin{tabular}{cc}
\hline $\begin{array}{c}\text { Concentración } \\
\text { (mg/litro) }\end{array}$ & $\begin{array}{c}\boldsymbol{\eta}_{\mathbf{r}} \\
\text { (mPa.s) }\end{array}$ \\
\hline 461 & 1,193 \\
948 & 1,470 \\
1355 & 1,750 \\
1851 & 2,187 \\
2294 & 2,517 \\
2808 & 2,930 \\
4614 & 5,587 \\
6782 & 9,305 \\
9138 & 17,360 \\
\hline
\end{tabular}




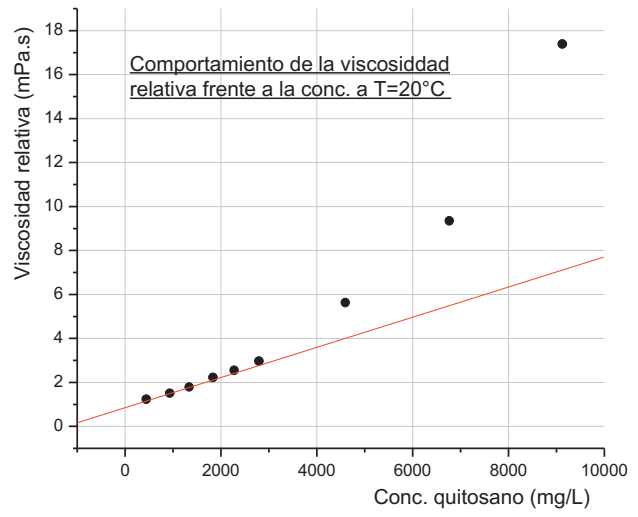

Figura 4. Comportamiento de la viscosidad relativa respecto de la concentración a $\mathrm{T}=20^{\circ} \mathrm{C}$.

Utilizando los datos de la tabla 2 se realizó una regresión lineal con las seis primeras concentraciones, resultando una dependencia lineal cuyo valor de $\mathrm{r}^{2}$ fue de 0,995 y valor de pendiente $7,55 \times 10^{-4}$. Como resultado quedó establecida una curva de calibración que responde a la ecuación lineal 6 y la figura 5. Esta dependencia lineal está enmarcada en el contexto de las soluciones ideales ${ }^{10,15}$.

$$
\eta_{r}=0,78505+7,54584 \times 10^{-4} C
$$

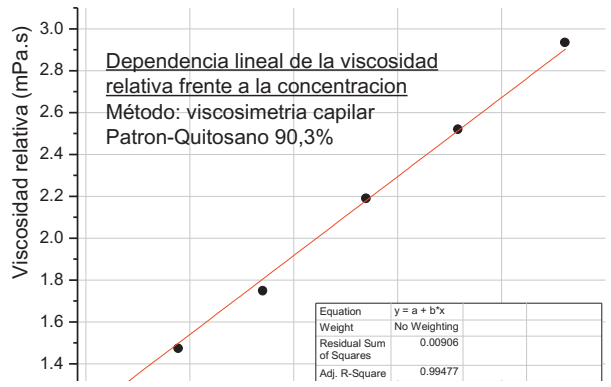

Figura 5. Curva de calibración de dependencia lineal de la viscosidad relativa frente a la concentración para soluciones diluidas a $20^{\circ} \mathrm{C}$.

Establecida la curva de calibración se procedió a realizar los ensayos para determinar la concentración desconocida de dos muestras problema de quitosano en polvo. En dichos ensayos se midieron los tiempos de flujo bajo las mismas condiciones de trabajo ya establecidas. Previamente se reprodujo la curva de calibración (figura 5) trabajada con el estándar de quitosano (patrón) como muestra la figura 6. 


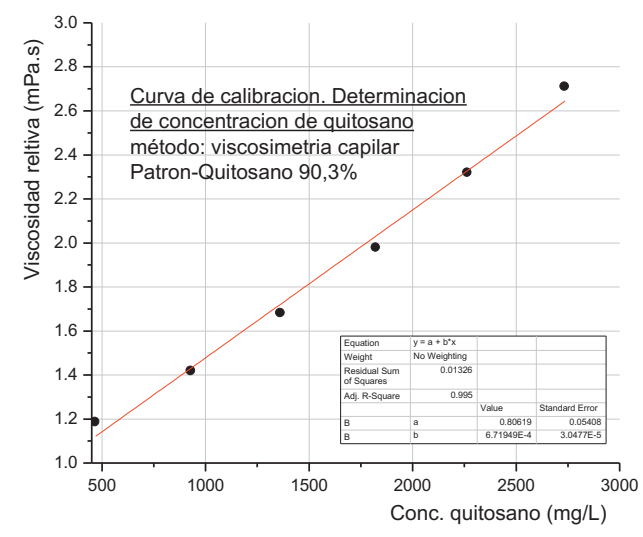

Figura 6. Reproducción de la curva de calibración empleada en la determinación de concentración de quitosano.

De la última gráfica realizada la regresión lineal se obtuvo la ecuación lineal 7.

$$
\eta_{r}=0,80422+6,7005 \times 10^{-4} C
$$

Medidos los tiempos de flujo del solvente y de las soluciones problema se procedió a calcular las viscosidades relativas, cuyos coeficientes reemplazados en la ecuación 7 se obtiene los valores de concentración de quitosano tal como muestra la tabla 4.

Tabla 3. Valores de concentración de quitosano obtenidos para la determinación de concentración desconocida de quitosano.

\begin{tabular}{cccccc}
\hline Muestra & $\mathbf{p H}$ & $\begin{array}{c}\boldsymbol{\eta}_{\mathbf{r}} \\
\text { (mPa.s) }\end{array}$ & $\begin{array}{c}\text { Concentración } \\
\text { preparada } \\
\text { (mg/litro) }\end{array}$ & $\begin{array}{c}\text { Concentración } \\
\text { determinada por } \\
\text { viscosimetría con } \\
\text { ecuación 7 } \\
\text { (mg/litro) }\end{array}$ & $\begin{array}{c}\text { Porcentaje } \\
\text { de } \\
\text { recuperación } \\
\text { (\%) }\end{array}$ \\
\hline M1 & 1,25 & 1,529 & 1147 & 1082 & 94,3 \\
M2 & 1,24 & 1,898 & 1643 & 1633 & 99,4 \\
\hline \multicolumn{2}{l}{ Porcentaje de recuperación promedio $=96,9 \%$}
\end{tabular}

Obtenidas las concentraciones se evidencia que ambos valores se ubican dentro del rango de concentraciones patrón establecido en la curva de calibración. 


\section{CONCLUSIONES}

Se ha puesto en evidencia una dependencia lineal de la viscosidad relativa respecto de la concentración en soluciones de quitosano en el rango de 460,53 mg/L a 2808,33 mg/L. Además, se ha corroborado que a mayor dilución del polímero existe una aproximación a las condiciones de soluciones ideales.

La dependencia más importante para una temperatura dada fue la de viscosidad relativa $\left(\eta_{\mathrm{r}}\right)$ frente a la concentración (concordante con la ecuación de Poiseuille) sustentada en la figura 5 con $r^{2}=0,995$.

Los resultados obtenidos para el análisis del contenido de quitosano en polvo fueron consistentes y equiparables con los de las fichas técnicas del producto.

Esta propuesta puede ser aplicable para la determinación de la pureza de quitosano en muestras en polvo.

\section{AGRADECIMIENTO}

Agradecemos al Laboratorio de Espectroscopía y Química Cuántica del Departamento de Fisicoquímica de la Facultad de Química e Ingeniería Química de la Universidad Nacional Mayor de San Marcos por la realización de los ensayos químicos. Asimismo, agradecer al Taller de Vidrio de la misma facultad por la fabricación del viscosímetro de Ostwald utilizado en el mencionado estudio.

\section{REFERENCIAS BIBLIOGRÁFICAS}

1. Ravi MN. A review of chitin and chitosan applications. React Funct Polym. 2000; 46: $1-27$.

2. Aranaz I, Mengíbar M, Harris R, Paños I, Miralles B, Acosta N, et al. Functional Characterization of Chitin and Chitosan. Curr Chem Biol. 2009; 3: 203-230.

3. Sorlier S, Denuzière A, Viton C, Domard A. Relation between the Degree of Acetylation and the Electrostatic Properties of Chitin and Chitosan. Biomacromolecules. 2001; 2: 765-772.

4. Tolaimate A, Desbrières J, Rhazi M, Alagui A, Vincendon M, Vottero P. On the influence of deacetylation process on the physicochemical characteristics of chitosan from squid chitin. Polymer. 2000; 41: 2463-2469.

5. de Alvarenga ES, de Oliveira CP, Bellato CR. An approach to understanding the deacetylation degree of chitosan. Carbohydr Polym. 2010; 80: 1155-1160.

6. Vårum KM, Ottфy MH, Smidsrфd O. Acid hydrolysis of chitosans. Carbohydr Polym. 2001; 46: 89-98. 
7. Noviov V. Acid Hydrolisis of Chitin and Chitosan. Russ J Appl Chem. 2004; 77 (3): 484-487.

8. Knill CJ, Kennedy JF, Mistry J, Miraftab M, Smart G, Groocock MR, et al. Acid hydrolysis of commercial chitosans. J Chem Technol Biotechnol. 2005; 80: 1291-1296.

9. Levine IN. Fisicoquímica. Volumen 2', quinta edición. Madrid, España: Editorial McGraw-Hill/Interamericana de España; 2004. 616.

10. Castellan GW. Fisicoquímica. Segunda edición. México D. F., México: Editorial Addison-Wesley Iberoamericana; 1987.

11. Park JW, Choi K-H. Acid-Base Equilibria and Related Properties of Chitosan. B Korean Chem Soc. 1983; 4(2): 68-72.

12. Desbrieres J. Viscosity of Semiflexible Chitosan Solutions: Influence of Concentration, Temperature, and Role of Intermolecular Interactions. Biomacromolecules. 2002; 3: 342-349.

13. El-Hefian EA, Khan RA, Yahaya AH. Study of the Parameters Affecting the Viscosity of Chitosan Solutions. J Chem Soc Pak. 2008; 30 (4): 529-531.

14. Chen RH, Tsaih ML. Effect of temperature on the intrinsic viscosity and conformation of chitosans in dilute $\mathrm{HCl}$ solution. Int J Biol Macromol. 1998; 23(2):135 $\square 141$.

15. Maron SH, Prutton CF. Fundamentos de fisicoquímica. Primera edición. México D.F., México: Editorial Limusa; 1980.

16. El- Hefian EA, Elgannoudi ES, Mainal A, Yahaya AH. Characterization of chitosan in acetic acid: Rheological and thermal studies. Turk J Chem. 2010; 34: 47-56.

17. Harding SE. The intrinsic viscosity of biological macromolecules. Progress in measurement, interpretation and application to structure in dilute solution. Prog Biophys Molec Biol. 1997; 68 (2/3): 207-262.

18. Kjфniksen AL, Nyströn B, Iversen C, Nakken T, Palmgren O, Tande T. Viscosity of Dilute Aqueous Solutions of Hydrophobically Modified Chitosan and Its Unmodified Analogue at Different Conditions of Salt and Surfactant Concentrations. Langmuir. 1997; 13: 4948-4952. 